\title{
BMJ Open Outpatient parenteral antimicrobial therapy (OPAT) versus inpatient care in the UK: a health economic assessment for six key diagnoses
}

\author{
Maria Dimitrova, ${ }^{1}$ Mark Gilchrist, ${ }^{2}$ R A Seaton (1) ${ }^{3,4}$
}

To cite: Dimitrova M, Gilchrist M, Seaton RA. Outpatient parenteral antimicrobial therapy (OPAT) versus inpatient care in the UK: a health economic assessment for six key diagnoses. BMJ Open 2021;11:e049733. doi:10.1136/ bmjopen-2021-049733

- Prepublication history and additional supplemental material for this paper are available online. To view these files, please visit the journal online (http://dx.doi.org/10.1136/ bmjopen-2021-049733).

Received 31 January 2021 Accepted 15 September 2021

Check for updates

(c) Author(s) (or their employer(s)) 2021. Re-use permitted under CC BY-NC. No commercial re-use. See rights and permissions. Published by BMJ.

${ }^{1}$ Scottish Health Technology Group, Healthcare Improvement Scotland, Edinburgh, UK

2Department of Infection/ Pharmacy, Imperial College Healthcare NHS Trust, London, UK

${ }^{3}$ Infectious Diseases, Queen Elizabeth University Hospital, Glasgow, UK

${ }^{4}$ Scottish Antimicrobial

Prescribing Group, Healthcare Improvement Scotland, Glasgow, UK

Correspondence to

Dr R A Seaton;

andrew.seaton@ggc.scot.nhs.uk

\section{ABSTRACT}

Objectives To compare costs associated with different models of outpatient parenteral antimicrobial therapy (OPAT) delivery with costs of inpatient (IP) care across key infection groups managed via OPAT in the UK.

Design A cost-minimisation design was used due to evidence of similarities in patient and treatment outcomes between OPAT and IP care. A bottom-up approach was undertaken for the evaluation of OPAT associated costs. The British Society of Antimicrobial Chemotherapy National Outcomes Registry System was used to determine key infection diagnoses, mean duration of treatment and most frequent antibiotics used.

Setting Several OPAT delivery settings were considered and compared with IP care.

Interventions OPAT models considered were OP clinic model, nurse home visits, self (or carer)-administration by a bolus intravenous, self-administration by a commercially prefilled elastomeric device, continuous intravenous infusion of piperacillin with tazobactam or flucloxacillin with elastomeric device as OP once daily and, specifically for bone and joint and diabetic foot infections, complex outpatient oral antibiotic therapies.

Results Base case and a range of scenario results showed all evaluated OPAT service delivery models to be less costly than IP stay of equivalent duration. The extent of savings varied by OPAT healthcare delivery models. Estimated OPAT costs as a proportion of IP costs were estimated at 0.23-0.53 (skin and soft-tissue infections), 0.34-0.46 (complex urinary tract infections), 0.23-0.51 (orthopaedic infections), $0.24-0.42$ (diabetic foot infections) $0.40-0.56$ (exacerbations of bronchiectasis) and $0.25-0.42$ (intra-abdominal infections). Partial or full complex oral antibiotic therapies in orthopaedic or diabetic foot infections costs were estimated to be $0.13-0.26$ of IP costs. Main OPAT costs were associated with staff time and antimicrobial medications.

Conclusions OPAT is a cost-effective use of National Health Service resources for the treatment of a range of infections in the UK in patients who can be safely managed in a non-IP setting.

\section{INTRODUCTION}

Outpatient parenteral antimicrobial therapy (OPAT) is a well-established and safe method of delivering intravenous antimicrobial

\section{STRENGTHS AND LIMITATIONS OF THIS STUDY}

$\Rightarrow$ First study to use a bottom-up costing approach applied across a range of infections and healthcare delivery models in outpatient parenteral antimicrobial therapy (OPAT) using UK-specific registry data.

$\Rightarrow$ First study to compare the cost of various models of care in OPAT with inpatient stay.

$\Rightarrow$ Base case results were consistent across a range of scenario analyses.

$\Rightarrow$ Assumed equivalence in OPAT and inpatient outcomes and between different OPAT models of care is associated with some uncertainty.

$\Rightarrow$ There are uncertainties in the quantification of some indirect OPAT costs (and their contribution to each OPAT model) and with inpatient bed day costs stay.

therapy to carefully selected patients in whom other aspects of care can be addressed without hospitalisation. ${ }^{12}$ The British Society for Antimicrobial Chemotherapy (BSAC) has developed good practice recommendations for safe adult and paediatric OPAT practice and clinical governance including promotion of antimicrobial stewardship. ${ }^{2}$ The recommendations acknowledged that a variety of OPAT delivery models were available and widely practised in the UK including home or clinic administration, administration by nurse or self/carer and administration via bolus or infusion. In 2015, BSAC launched the National Outcomes Registry System (NORS) for UK OPAT services in order to describe the OPAT landscape (including infections treated and antimicrobials used) and to enable participating centres to benchmark practice and outcomes. ${ }^{3}$ Despite popularity and growth in OPAT practice in the UK, funding/reimbursement of OPAT has been inconsistent and as a result OPAT has not been uniformly established. A previous publication attempted to describe cost-effectiveness of OPAT in a tariff based UK health model for a single condition (cellulitis). ${ }^{4}$ Herein, we 
Table 1 Average duration and total number of treatment episodes in OPAT for six categories of infection

\begin{tabular}{llll} 
Condition & $\begin{array}{l}\text { Average } \\
\text { duration } \\
\text { (days) }\end{array}$ & Total no & Source \\
\hline SSTI & 6.4 & 7371 & \multicolumn{2}{l}{$\begin{array}{l}\text { NORS 2015-19 } \\
\text { Complex UTI }\end{array}$} & 7.0 & 1896 & \\
\cline { 1 - 2 } (UK)
\end{tabular}

NORS, national outcomes registry system; OPAT, outpatient parenteral antimicrobial therapy; SSTI, skin and soft-tissue infections; UTI, urinary tract infections.

estimate OPAT-related costs for a number of key indicator conditions utilising different established OPAT delivery models and compare with reference inpatient costs. The analysis also set out to take into account evolution of practice with greater use of supervised complex oral antibiotic therapies within the OPAT setting. ${ }^{5}$

\section{METHODS}

The costs of six OPAT healthcare delivery models were estimated and compared against the cost of equivalent duration of inpatient stay for six key infection categories typically managed via OPAT in the UK. Based on available clinical evidence and clinical expert opinion, equivalence in patient outcomes was assumed for OPAT and inpatient stay as well as for the different models of healthcare delivery in OPAT. ${ }^{6-8}$ A cost-minimisation analysis was therefore deemed appropriate. A list of detailed costing model assumptions primarily based on the BSAC updated good practice recommendations ${ }^{2}$ and clinical expert opinion is available in online supplemental materials (online supplemental table 1.

\section{NORS data}

The cost-minimisation analysis used 5 years of retrospective data relating to 21632 adult treatment episodes collected from 57 OPAT centres (44 in England, 5 in Scotland, 4 in Wales and 4 in Northern Ireland), that all reported to $\mathrm{NORS}^{3}$ (table 1). In the cost-minimisation model, patients were treated in OPAT for one of six broadly defined infection categories, representing approximately $82 \%$ of primary OPAT diagnoses recorded in NORS. These were infections requiring short-term (up to 7 days) antimicrobial treatment such as skin and softtissue infections including cellulitis (SSTI) and complex urinary tract infections (UTI) (including drug-resistant lower UTI and pyelonephritis) or longer-term antimicrobial treatment such as bone and joint (including spinal, native osteomyelitis and orthopaedic metal work associated infections), diabetic foot infections (both osteomyelitis and complex soft-tissue infections), bronchiectasis and intra-abdominal infections (including liver abscess and other abdominal and pelvic infections) (online supplemental table 2).

\section{OPAT healthcare delivery models}

The OPAT models considered were based on those currently practised in the UK: outpatient clinic model, nurse home visits, self (or carer)-administration by a bolus intravenous, self-administration via an elastomeric device including continuous intravenous infusion (CIVI) of piperacillin with tazobactam or flucloxacillin and (for bone and joint or diabetic foot infections) complex outpatient antibiotic therapy. Only suitable healthcare models were considered for the treatment of each of the six conditions in OPAT.

The care pathway for the outpatient clinic model requires a patient to travel daily to the OPAT unit following an initial assessment in hospital. At the clinic, an OPAT nurse prepares and administers antimicrobial medication intravenously by bolus intravenous. The nurse home visit model entails a specialist nurse (or a community-based nurse) travelling to the patient's home instead.

The self-administration model of OPAT care requires the patient (or carer) to administer the antimicrobials at home, without the need to travel to hospital or for a nurse to visit on a daily basis. It is assumed that prior to commencing antimicrobial self-administration, patients (or their carers) receive on average three training sessions (60 min each with a nurse) on how to safely prepare and administer their medication through a peripherally inserted central catheter (PICC) line using a bolus intravenous. It is further assumed that only one training session is required for patient/carer administration of preprepared medication via an elastomeric home infusion device which only needs to be connected to and disconnected from the PICC line correctly. The patient is discharged with the necessary consumables (eg, a leaflet of instructions, syringes, needles, $70 \%$ alcohol wipes, $0.9 \%$ sodium chloride ampoules, sharps bin, vials with medication powder for infusion, etc) and is given a contact number related to the OPAT service for use if any problems occur out of hours. On the occasion where an elastomeric pump is used for self-administration, these are commercially prefilled devices due to safety issues regarding the process of reconstituting drugs into elastomeric devices outside of an aseptic unit and the associated reduction in shelf life (approximately 24 hours). A CIVI model of service delivery in OPAT involves reconstitution of drugs into elastomeric devices by hospital staff with piperacillin with tazobactam or flucloxacillin on the same day of administration with the patient visiting clinic once daily. ${ }^{9} 10$

Patients referred to OPAT with bone and joint infections (eg, diabetic foot and orthopaedic infections) are increasingly considered for suitability for discharge on supervised complex oral therapies as an alternative to 'traditional' OPAT intravenous therapy. Suitable patients may be commenced directly onto supervised 
oral antimicrobial treatment which frequently requires enhanced monitoring due to potentially serious adverse events (eg, linezolid or combination oral antibiotic regimens) ${ }^{5}$

In all OPAT models where treatment is required for $>1$ week patients will also attend the OPAT clinic once weekly as a minimum and management/progress will be reviewed via a weekly virtual multidisciplinary meeting. ${ }^{2}$

\section{Antimicrobials}

Antimicrobial medications with a lower frequency of administration compared with inpatient care are primarily selected for use in OPAT. In the analysis, the type and distribution of antimicrobial medicines are based on clinical expert opinion and the most prevalent agents used within the NORS database (online supplemental table 3).

Antimicrobial medicines which require more than once daily administration (eg, piperacillin with tazobactam, flucloxacillin, temocillin, ceftazidime and meropenem) would be primarily administered by the patient or carer. However, if an antibiotic is stable to degradation in the outpatient setting (piperacillin with tazobactam and flucloxacillin), patients may receive the antibiotic via a 24-hour continuous infusion (CIVI) via an elastomeric device as an outpatient requiring once daily attendance. All other antimicrobial medicines where data on stability are either not available or are not compatible with 24-hour infusion may be administered with bolus intravenous or 30 min intravenous infusion.

\section{Rehospitalisation}

The cost-minimisation model assumed hospital readmission to occur in $6.4 \%$ of treatment episodes in OPAT. ${ }^{7}$ Rehospitalised patients were assumed to only spend $50 \%$ of the duration of their treatment episode in OPAT.

\section{Costs}

Costs considered in the analysis included specialist staff time (specialist nurses, infectious diseases consultant and specialist antimicrobial/clinical pharmacist), antimicrobial medications, elastomeric infusion devices (empty or commercially prefilled), consumables, laboratory tests and the cost of travel to and from the OPAT clinic where necessary (online supplemental tables 4-6). Additionally, costs of rehospitalisation for patients in OPAT have been included. A daily 'overhead' cost per patient to account for administration and support costs of using a healthcare service consistent with published literature has also been assumed in the analysis. ${ }^{8}$

Costs were obtained from various sources. These included the Personal and Social Services Research Unit, ${ }^{11}$ British National Formulary (BNF) ${ }^{12}$ drugs and pharmaceutical electronic market information tool (eMIT),${ }^{13}$ National Procurement Scotland, Information Services Division (ISD) Scotland cost book ${ }^{14}$ and National Health Service (NHS) England reference costs. ${ }^{15}$

An appropriate healthcare resource group code ${ }^{15}$ was identified for each diagnosis to account for costs of inpatient care (online supplemental table 7). Costs were based on a weighted average of excess bed day costs as elective and non-elective inpatient stay across various severity levels. No potential additional hospital costs were included.

\section{Patient and public involvement}

No patient involved.

\section{RESULTS}

Base case results from the cost-minimisation analysis are presented as estimated average cost per treatment episode in inpatient care and models of OPAT healthcare delivery, as well as cost of OPAT as a percentage of the estimated cost of inpatient stay (tables 2 and 3). Total costs of models of care and savings associated with OPAT across all conditions included within the NORS data set are shown in online supplemental table 8.

\section{Infections requiring short-term treatment \\ Skin and soft-tissue infections}

In the cost-minimisation analysis, patients attending once daily for the duration of antimicrobial treatment, were primarily treated with intravenous ceftriaxone, but also with teicoplanin and daptomycin (for beta-lactam allergy). Treatment with the same medicines was assumed in the specialist nurse visit model. A small number of patients could also be treated with dalbavancin $1 \mathrm{~g}$ as a one-off dose, consistent with clinical practice for patients unable to attend for daily treatment. Patients who were offered an elastomeric home infusion device were primarily treated with ceftriaxone once daily and less often with flucloxacillin as 24 hours (CIVI). Dependant on the OPAT service delivery model, the cost of treating patients with SSTI was estimated in the range of $23 \%-51 \%$ of the cost of inpatient stay for the equivalent duration of treatment.

\section{Complex UTIs}

In the analysis, patients were primarily treated with ertapenem $(90 \%)$ and a minority of patients were treated with temocillin. It was assumed that patients attending daily or treated by a nurse at home were only given ertapenem due to twice daily dosing of temocillin. Both antibiotics can be self-administered using either short infusion or intravenous bolus, respectively. No patients were assumed to be treated using home infusion elastomeric devices. Dependant on the OPAT service delivery model, the cost of treating patients with complex UTI was estimated in the range of $34 \%-46 \%$ of the cost of inpatient stay for the equivalent duration of treatment.

\section{Infections requiring longer-term treatment Orthopaedic infections}

Patients with orthopaedic infections were assumed to be treated once daily with ceftriaxone, teicoplanin or ertapenem in the outpatient visit, nurse home visit and selfadministration OPAT service delivery models. Ceftriaxone was the only antimicrobial used for self-administration via 


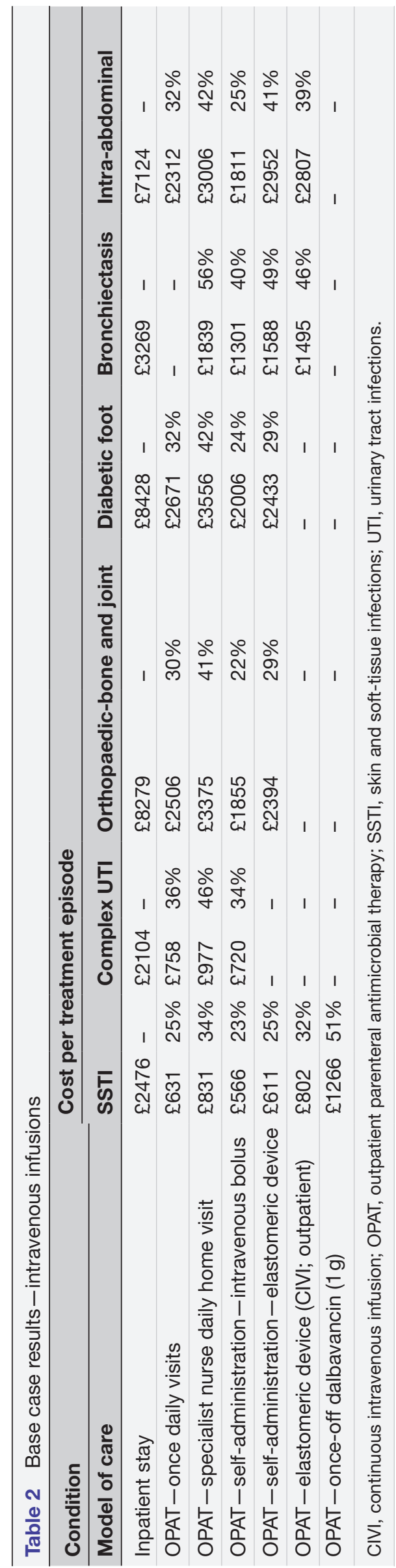

commercially prefilled elastomeric device. An analysis is also presented where patients were treated with oral therapies only or assuming intravenous to oral switch at different time points during supervised outpatient therapy. Dependant on the OPAT service delivery model (excluding oral therapies), the cost of treating patients with orthopaedic infections was estimated in the range of $22 \%-41 \%$ of the cost of inpatient stay for the equivalent duration of treatment. Additionally, a movement in treatment practice from intravenous to oral antibiotics could results in treatment cost of $13 \%-24 \%$ of the cost of inpatient stay, dependent on how early a patient is switched to oral therapy.

\section{Diabetic foot infections}

Similarly to orthopaedic infections, patients with diabetic foot infections were assumed to be treated with ceftriaxone, ertapenem and teicoplanin, although the proportion of patients treated with each agent varied. All antimicrobial medicines are suitable for once daily administrations. The cost of treatment in OPAT was estimated in the range of $22 \%-42 \%$ of the cost of inpatient stay for the equivalent duration of treatment. Additionally, a movement in treatment practice from intravenous to oral antibiotics could results in treatment cost of $13 \%-26 \%$ of the cost of inpatient stay, dependent on how early a patient is switched to oral therapy.

\section{Bronchiectasis}

In the analysis, patients were assumed to be treated with either ceftazidime, meropenem or piperacillin with tazobactam. All medications are suitable for intravenous selfadministration with either a bolus or $30 \mathrm{~min}$ infusion. Only piperacillin with tazobactam was assumed to be administered with an elastomeric device via a 24-hour continuous infusion in an outpatient setting (CIVI). The per-episode estimated cost for the same treatment plan with a visiting nurse is also presented. Since all of these medications require more than once daily administration or continuous daily administration, the general outpatient daily visits and nurse home visits OPAT service models are highly unlikely to be used in clinical practice so the results from CIVI models are presented instead. The estimated per treatment episode cost is in the range of $40 \%-56 \%$ of the cost of inpatient stay and varies with selected OPAT model of care.

\section{Intra-abdominal infections}

In the cost-minimisation analysis, patients were primarily treated with ertapenem or piperacillin with tazobactam. The latter was assumed to be used only in patients who were suitable for self-administration or attend daily for change of elastomeric device (CIVI). Patients who visit daily for intravenous infusions or receive nurse visits were assumed to only be treated with ertapenem. The estimated per treatment episode cost is in the range of $25 \%-42 \%$ of the cost of inpatient stay and varies with selected OPAT model of care. 
Table 3 Base case results - oral antimicrobials for orthopaedic and diabetic foot infections

\begin{tabular}{llrr}
\hline Condition & \multicolumn{2}{l}{ Orthopaedic/bone and joint } & Diabetic foot \\
\hline Inpatient stay & $£ 8279$ & & $£ 8428$ \\
\hline OPAT-oral 100\% & $£ 1114$ & $13 \%$ & $£ 1089$ \\
\hline OPAT-oral 25\%; 75\% IV & $£ 2009$ & $24 \%$ & $£ 2161$ \\
\hline OPAT-oral 50\%; 50\% IV & $£ 1710$ & $21 \%$ & $26 \%$ \\
\hline OPAT-oral 75\%; 25\% IV & $£ 1410$ & $17 \%$ & $22 \%$ \\
\hline
\end{tabular}

IV, intravenous; OPAT, outpatient parenteral antimicrobial therapies.

\section{Scenario analyses}

In scenario analyses described in table 4, certain modelling assumptions were challenged to assess impact on results in the outpatient setting model. Detailed scenario analysis results are provided in online supplemental table 9.

Results from scenarios 1 and 2 (costs ranging between $21 \%$ and $34 \%$ of inpatient costs) are consistent, although lower than base case $(25 \%-44 \%)$ across all infection categories due to the higher cost per bed day generally associated with an infectious disease unit (IDU).

Given the modelling approach, one of the uncertainties comes from the extent of model-specific per-patient overhead costs (scenario 3 ). The uncertainty is especially relevant to the outpatient clinic model. One study ${ }^{16}$ reported overall overhead and support costs in the outpatient department of their IDU to be $44.8 \%$ of total costs excluding re-hospitalisation. When the equivalent assumption was made in the outpatient clinic model, the cost of treatment episode in OPAT did not increase over $52 \%$ of the cost of inpatient stay, except for bronchiectasis $(77 \%)$ which is treated with CIVI with elastomeric device as an outpatient visit once daily in the analysis which is generally associated with higher cost.

There are uncertainties around the source of cost for linezolid for the oral treatment of orthopaedic and diabetic foot infections (scenario 4). The cost reported in the $\mathrm{BNF}^{12}$ is substantially higher than what clinicians have advised and also reported in the $\mathrm{eMIT}^{13}$ which has been used in the base case analysis. Using the higher cost of linezolid results in the cost of oral therapies increasing from $13 \%$ to $21 \%$ of the cost of inpatient stay.

Additionally, variations in the treatment protocol with dalbavancin seems to have the highest impact on costs of treatment of SSTIs in OPAT due to the high medicine acquisition cost (scenarios 6 and 7). Treatment with dalbavancin can be as costly as $74 \%-76 \%$ of the cost of inpatient stay.

\section{DISCUSSION}

Although OPAT has been developing in the UK over the last 25 years, and despite the clinical benefits of avoiding hospitalisation and keeping care closer to home, there

Table 4 Scenarios: OPAT outpatient versus inpatient stay

\begin{tabular}{|c|c|c|}
\hline & Scenario & Base case \\
\hline 1 & $\begin{array}{l}\text { Using cost of inpatient care in infectious disease unit } \\
\text { (ISD Scotland })^{14}\end{array}$ & Using condition-specific healthcare resource group costs ${ }^{15}$ \\
\hline 2 & $\begin{array}{l}\text { Using ISD Scotland }{ }^{14} \text { cost for outpatient appointments } \\
\text { and inpatient stay (IDU) }\end{array}$ & $\begin{array}{l}\text { Using microcosting of nurse and consultant outpatient } \\
\text { appointments }\end{array}$ \\
\hline 4 & $\begin{array}{l}\text { Using } \mathrm{BNF}^{12} \text { as a source for the cost of linezolid } \\
\text { (orthopaedic and diabetic foot infections) }\end{array}$ & $\begin{array}{l}\text { Using } \mathrm{eMIT}^{13} \text { as a source for the cost of linezolid (orthopaedic } \\
\text { and diabetic foot infections) }\end{array}$ \\
\hline \multicolumn{3}{|c|}{ Skin and soft-tissue infections only } \\
\hline 5 & Including the cost of consultant time & Nurse-led condition; no consultant time \\
\hline
\end{tabular}

BNF, British National Formulary; eMIT, electronic market information tool; IDU, infectious disease unit; ISD, information services division; OPAT, outpatient parenteral antimicrobial therapy. 
remains wide variation in OPAT availability and inconsistency in funding/commissioning of services in the UK. It is possible that OPAT may be perceived as an additional healthcare cost and this could be a significant barrier to more systematic support for service development. This study therefore set out to systematically detail OPAT costs and compare with inpatient costs for key OPAT-treated conditions. To our knowledge, a detailed costing of various OPAT healthcare delivery models in a UK setting has not been published before. Nevertheless, results are consistent with previous studies where overall cost of OPAT have been reported and/or compared with the cost of inpatient care. ${ }^{616-18}$ Key findings are that the self (or care)-administration model of care was associated with the lowest cost and nurse home visits generally had the highest estimated cost per treatment episode across all conditions evaluated. From all available OPAT models for patients with SSTIs, treatment with a single dose of dalbavancin was estimated to have the highest cost. However, only a small proportion of patients (an estimated 5\%) are expected to be treated with dalbavancin in clinical practice. As expected, oral therapies were the lowest cost treatment option for patients with orthopaedic (bone and joint) or diabetic foot infection. If patients are switched from intravenous as outpatient to oral therapies at least half way through their treatment duration, the results show the cost of treatment episode to be lower than the cost of self-administering intravenous antimicrobials for the whole treatment duration.

The cost-minimisation analysis found all OPAT service delivery models to be consistently associated with lower cost than inpatient stay of equivalent duration across a range of conditions in the UK NHS. The analysis shows the potential of OPAT to provide quality healthcare for suitable patients in an outpatient setting at a fraction of the cost of inpatient care. When the four most frequently used OPAT models of care (outpatient clinic, nurse home visit self (carer)-administration (bolus intravenous or elastomeric device) are considered individually, using NORS UK data, ${ }^{3}$ the 5 year estimated savings to the UK NHS associated with treatment in OPAT was found to be in the range of $£ 60-77$ million (online supplemental table 9). It should be noted that these data only relate to 57 OPAT services and many have not contributed data consistently for all 5 years (data not shown). The estimated cost savings to the NHS through systematic roll out of OPAT therefore is likely to be considerably higher.

Strengths of the presented analysis include a bottom-up costing approach applied across a range of infections, and healthcare delivery models in OPAT using UK-specific registry data. Furthermore, each of these estimated costs were compared against the cost of inpatient care in a UK setting and a comprehensive list of scenario analyses showed consistency with base case findings. However, the study also had some limitations. The assumption of equivalence in patient and treatment outcomes for OPAT and inpatient care as well as among various models of care in OPAT is based on published systematic reviews ${ }^{6-8}$ but direct comparative evidence is lacking. Furthermore, there was one study ${ }^{8}$ to suggest that a specialist nurse visit model is generally associated with better outcomes compared with other healthcare delivery models in OPAT. A published source was used for the cost of inpatient stay which is inconsistent with the bottom-up costing approach undertaken for the cost of OPAT. ${ }^{15}$ The assumption of cost of bed day in the analysis to be equivalent to the cost of excess bed days, as reported in NHS England Reference costs,${ }^{15}$ is also associated with uncertainties due to the structure of the reimbursement system in NHS England (eg, the presence of trim points). Nevertheless, this is considered to be the most suitable published source of costs of inpatient stay as it provides cost estimates for each of the six infection categories in the analysis. Lastly, the estimated average costs per treatment episode in OPAT aim to reflect existing OPAT services and thus set-up and implementation costs have not been included.

It is important to note that most OPAT services will not rely on one particular delivery model and that individual patient factors such as, ability to self-administer or to attend the OPAT clinic, and choice of antimicrobial, will be the major determinants of the delivery model used. The data presented necessarily incorporate multiple variables to allow for variation based on patient need.

OPAT is primarily about delivering high quality patient centred care closer to home while avoiding inherent risks associated with hospitalisation. These positive health economic findings should be utilised by OPAT clinician/ practitioners, healthcare managers and policy makers alongside the already powerful clinical effectiveness and patient safety data to drive further OPAT development in the UK.

\section{Twitter R A Seaton @raseaton66}

Acknowledgements This study was initiated on behalf of the British Society of Antimicrobial Chemotherapy UK OPAT initiative in conjunction with the Scottish Antimicrobial Prescribing group and is part of a broader OPAT health technology assessment performed by the Scottish Health Technology Group, Healthcare Improvement Scotland. Specific thanks to: Dr Felicity Drummond for access to and summary data from the National UK OPAT Registry and to the 57 UK OPAT services who have contributed to the NORS database; Healthcare Improvement Scotland: Jenny Harbour for summarising relevant clinical evidence, Dr Carolyn Sleith for undertaking a literature search, Meryl Heggeland for checking to validity of the economic methods and results, Edward Clifton for general support with the project; for providing clinical advice: from NHS Lothian: Dr Claire Mackintosh and Sharon Watson; from NHS Tayside: Dr Busi Mooka, Dr Jorge Rojas Marcos and Gain Menzies; from NHS Fife: Dr David Griffith.

Contributors MD: responsible for developing the economic model, completing the health economic analysis and preparation of the manuscript; RAS and MG: responsible for conceiving the concept of the study, advising on the development of the economic model and preparation of the manuscript. All authors have approved the final version of the manuscript.

Funding The authors have not declared a specific grant for this research from any funding agency in the public, commercial or not-for-profit sectors.

Competing interests None declared.

Patient consent for publication Not applicable.

Provenance and peer review Not commissioned; externally peer reviewed.

Data availability statement No data are available. All data are included within the submission. 
Supplemental material This content has been supplied by the author(s). It has not been vetted by BMJ Publishing Group Limited (BMJ) and may not have been peer-reviewed. Any opinions or recommendations discussed are solely those of the author(s) and are not endorsed by BMJ. BMJ disclaims all liability and responsibility arising from any reliance placed on the content. Where the content includes any translated material, BMJ does not warrant the accuracy and reliability of the translations (including but not limited to local regulations, clinical guidelines, terminology, drug names and drug dosages), and is not responsible for any error and/or omissions arising from translation and adaptation or otherwise.

Open access This is an open access article distributed in accordance with the Creative Commons Attribution Non Commercial (CC BY-NC 4.0) license, which permits others to distribute, remix, adapt, build upon this work non-commercially, and license their derivative works on different terms, provided the original work is properly cited, appropriate credit is given, any changes made indicated, and the use is non-commercial. See: http://creativecommons.org/licenses/by-nc/4.0/.

ORCID iD

R A Seaton http://orcid.org/0000-0002-2509-0597

\section{REFERENCES}

1 Chapman ALN. Outpatient parenteral antimicrobial therapy. BMJ 2013;346:f1585.

2 Chapman ALN, Patel S, Horner C, et al. Updated good practice recommendations for outpatient parenteral antimicrobial therapy (OPAT) in adults and children in the UK. JAC Antimicrob Resist 2019;1:dlz026.

3 British Society for Antimicrobial Chemotherapy. National outcomes registry system (NORS), c2019. Available: https://www.e-opat.com/ opat-registry/

4 Jones GR, Cumming DVE, Honeywell G, et al. How is income generated by outpatient parenteral antibiotic treatment (OPAT) in the UK? analysis of payment tariffs for cellulitis. J Antimicrob Chemother 2015;67:1236-40.

5 Seaton RA, Ritchie ND, Robb F, et al. From 'OPAT' to 'COpAT': implications of the OVIVA study for ambulatory management of bone and joint infection. J Antimicrob Chemother 2019;74:2119-21.

6 Mitchell ED, Czoski Murray C, Meads D, et al. Clinical and costeffectiveness, safety and acceptability of c ommunity i ntra $v$ enous a ntibiotic s ervice models: CIVAS systematic review. BMJ Open 2017;7:e013560.

7 Sriskandarajah S, Hobbs J, Roughead E, et al. Safety and effectiveness of 'hospital in the home' and 'outpatient parenteral antimicrobial therapy' in different age groups: A systematic review of observational studies. Int J Clin Pract 2018:e13216 (published Online First: 2018/06/20).

8 Minton J, Murray CC, Meads D. The community intravenous antibiotic study (CIVAS): a mixed-methods evaluation of patient preferences for and cost-effectiveness of different service models for delivering outpatient parenteral antimicrobial therapy. NIHR Journals Library Health Services and Delivery Research 2017;02:02.

9 Allwood MC, Stonkute D, Wallace A, et al. Assessment of the stability of citrate-buffered flucloxacillin for injection when stored in two commercially available ambulatory elastomeric devices: INfusor LV (Baxter) and Accufuser (Woo young medical): a study compliant with the NHS yellow cover document (yCD) requirements. Eur J Hosp Pharm 2020;27:90-4.

10 Jamieson C, Ozolina L, Seaton RA, et al. Assessment of the stability of citrate-buffered piperacillin/tazobactam for continuous infusion when stored in two commercially available elastomeric devices for outpatient parenteral antimicrobial chemotherapy: a study compliant with the NHS yellow cover document requirements. Eur J Hosp Pharm 2020. doi:10.1136/ejhpharm-2020-002340. [Epub ahead of print: 2509 2020].

11 PSSRU. Unit costs of health and social care, 2020. Available: https:// www.pssru.ac.uk/project-pages/unit-costs/

12 BNF. BNF: British National formulary, 2020. Available: https://bnf. nice.org.uk

13 Department of Health. Drugs and pharmaceutical electronic market information tool (eMIT), 2020. Available: https://www.gov.uk/ government/publications/drugs-and-pharmaceutical-electronicmarket-information-emit\#: :text=National\%20Health\%20Service-, Drugs\%20and\%20pharmaceutical\%20electronic\%20market\% 20information\%20tool\%20(eMIT),generic\%20drugs\%20and\% 20pharmaceutical\%20products

14 Public Health Scotland. Cost books 2018/19, 2019. Available: https://www.isdscotland.org/Health-Topics/Finance/Costs/DetailedTables/

15 NHS Digital. Reference costs: 2019-20 reference costs collection, 2020. Available: https://digital.nhs.uk/data-and-information/datacollections-and-data-sets/data-collections/reference-costs

16 Chapman ALN, Dixon S, Andrews D, et al. Clinical efficacy and costeffectiveness of outpatient parenteral antibiotic therapy (OPAT): a UK perspective. J Antimicrob Chemother 2009;64:1316-24.

17 Durojaiye OC, Bell H, Andrews D, et al. Clinical efficacy, cost analysis and patient acceptability of outpatient parenteral antibiotic therapy (OPAT): a decade of Sheffield (UK) OPAT service. Int J Antimicrob Agents 2018;51:26-32.

18 Seaton RA, Johal S, Coia JE, et al. Economic evaluation of treatment for MRSA complicated skin and soft tissue infections in Glasgow hospitals. Eur J Clin Microbiol Infect Dis 2014;33:305-11. 